\title{
LOWER BOUNDS FOR OPERATORS ON GRADED LIE GROUPS
}

\author{
VÉRONIQUE FISCHER AND MICHAEL RUZHANSKY
}

\begin{abstract}
RÉSUMÉ. In this note we present a symbolic pseudo-differential calculus on any graded (nilpotent) Lie group and, as an application, a version of the sharp Gårding inequality. As a corollary, we obtain lower bounds for positive Rockland operators with variable coefficients as well as their Schwartzhypoellipticity.
\end{abstract}

\section{INTRODUCTION}

In this note we present a symbolic pseudo-differential calculus on any graded Lie group. As applications, we obtain a version of the sharp Gårding inequality and results on the Schwartz hypoellipticity for operators in this context.

In the usual Euclidean setting, the positivity of the full symbol is required for the sharp Gårding inequality as well as, for instance, for the Fefferman-Phong inequality. This contrasts with the Gårding or Melin-Hörmander inequalities, for example, where knowing the principal (or subprincipal) symbol is sufficient. Thus the latter inequalities can be proved on manifolds using the standard Hörmander theory of pseudo-differential operators together with the usual Kohn-Nirenberg quantisation on $\mathbb{R}^{n}$. Since the geometric control of the full symbol of an operator is impossible with these tools in general, the study of sharp Gåring inequalities appears limited. However, the sharp Gårding inequality on compact Lie groups was recently established in 13. This approach uses the notion of a full matrix-valued symbol defined in terms of the representation theory of the group. In this note we explain how we follow the same strategy in the case of the Heisenberg group or more general nilpotent Lie groups.

The pseudo-differential calculus that we define is different from the several pseudo-differential calculi already developed on the Heisenberg group : see e.g. Taylor [15] and [16] for symbol classes coming from standard Hörmander classes though the exponential mapping; Bahouri, Fermanian-Kammerer and Gallagher 1] for classes defined in terms of explicit formulae coming from the Schrödinger representation; or Beals and Greiner [2] or Ponge [1] for different types of analysis on the Heisenberg manifolds. On more general nilpotent groups, Christ, Geller, Glowacki and Polin [4 proposed an approach to pseudo-differential operators, however based on the properties of kernels and not on a symbolic calculus. Following Ruzhansky and Turunen [14] and [12, we define symbol classes directly on the group. As such, our approach can be extended for general graded nilpotent Lie

Date: February, 2013.

1991 Mathematics Subject Classification. Primary 35S05; Secondary 43A80.

Key words and phrases. harmonic analysis, nilpotent Lie groups, pseudo-differential operators. 
groups, and by developing the symbolic calculus and the Friedrichs approximation on the group, we obtain the corresponding sharp Gårding inequality and Schwartz hypoellipticity results.

While the symbol classes in [12] are based on the spectral theory of the LaplaceBeltrami operator, here, it is not available and it becomes natural to use the sub-Laplacian on stratified groups or more general Rockland operators on graded groups. Moreover, surpassing [13], since a dilation structure is present, we establish the sharp Gårding inequality for suitable $(\rho, \delta)$ classes of operators by establishing a Calderón-Vaillancourt type theorem in this context. This is, in fact, the best known lower bound available in the $(\rho, \delta)$-setting already on $\mathbb{R}^{n}$.

The appearing operators are Calderón-Zygmund in the sense of Coifman and Weiss [5, ch. III], so that $L^{p}$ results follow as well. In Section 2 we fix the notation concerning Lie groups that we are working on. In Section 3 we formulate the results.

\section{Preliminaries}

Let us first briefly recall the necessary notions and set some notation. In general, we will be concerned with graded Lie groups $G$ which means that $G$ is a connected and simply connected Lie group (of step $s>1$ ) with the gradation of its Lie algebra

$\mathfrak{g}$ given by $\mathfrak{g}=\underset{\ell=1}{\oplus} \mathfrak{g}_{\ell}$ with $\left[\mathfrak{g}_{\ell}, \mathfrak{g}_{\ell^{\prime}}\right] \subset \mathfrak{g}_{\ell+\ell^{\prime}}$ for any $\ell, \ell^{\prime} \in \mathbb{N}$, where the $\mathfrak{g}_{\ell}, \ell=1,2, \ldots$ are vector subspaces of $\mathfrak{g}$, almost all equal to $\{0\}$. This implies that the group $G$ is nilpotent. If the whole $\mathfrak{g}$ is generated by $\mathfrak{g}_{1}$ in this way, the group $G$ is said to be stratified.

Let $\left\{X_{1}, \ldots X_{n_{1}}\right\}$ be a basis of $\mathfrak{g}_{1}$ (this basis is possibly reduced to $\{0\}$ ), let $\left\{X_{n_{1}+1}, \ldots, X_{n_{1}+n_{2}}\right\}$ a basis of $\mathfrak{g}_{2}$ and so on, so that we obtain a basis $X_{1}, \ldots, X_{n}$ of $\mathfrak{g}$ adapted to the gradation. Via the exponential mapping $\exp _{G}: \mathfrak{g} \rightarrow G$, we identify the points $\left(x_{1}, \ldots, x_{n}\right) \in \mathbb{R}^{n}$ with the points $x=\exp _{G}\left(x_{1} X_{1}+\cdots+x_{n} X_{n}\right)$ in $G$. This leads to a corresponding Lebesgue measure on $\mathfrak{g}$ and the Haar measure $d x$ on the group $G$. We define the spaces of Schwartz functions $\mathcal{S}(G)$ and tempered distributions $\mathcal{S}^{\prime}(G)$ of the group $G$ as those on $\mathbb{R}^{n}$. The coordinate function $x=$ $\left(x_{1}, \ldots, x_{n}\right) \in G \mapsto x_{j} \in \mathbb{R}$ is denoted by $x_{j}$. More generally we define for every multi-index $\alpha \in \mathbb{N}_{0}^{n}, x^{\alpha}:=x_{1}^{\alpha_{1}} x_{2}^{\alpha_{2}} \ldots x_{n}^{\alpha_{n}}$, as a function on $G$. Similarly we set $X^{\alpha}=X_{1}^{\alpha_{1}} X_{2}^{\alpha_{2}} \cdots X_{n}^{\alpha_{n}}$ in the universal enveloping Lie algebra of $\mathfrak{g}$.

For any $r>0$, we define the linear mapping $D_{r}: \mathfrak{g} \rightarrow \mathfrak{g}$ by $D_{r} X=r^{\ell} X$ for every $X \in \mathfrak{g}_{\ell}, \ell \in \mathbb{N}$. Then the Lie algebra $\mathfrak{g}$ is endowed with the family of dilations $\left\{D_{r}, r>0\right\}$ and becomes a homogeneous Lie algebra in the sense of $[8]$. The weights of the dilations are the integers $v_{1}, \ldots, v_{n}$ given by $D_{r} X_{j}=r^{v_{j}} X_{j}, j=1, \ldots, n$. The associated group dilations are defined by

$$
r \cdot x:=\left(r^{v_{1}} x_{1}, r^{v_{2}} x_{2}, \ldots, r^{v_{n}} x_{n}\right), \quad x=\left(x_{1}, \ldots, x_{n}\right) \in G, r>0 .
$$

In a canonical way this leads to the notions of homogeneity for functions and operators. For instance the degree of homogeneity of $x^{\alpha}$ and $X^{\alpha}$, viewed respectively as a function and a differential operator on $G$, is $[\alpha]=\sum_{j} v_{j} \alpha_{j}$. Indeed, let us recall that a vector of $\mathfrak{g}$ defines a left-invariant vector field on $G$ and more generally that the universal enveloping Lie algebra of $\mathfrak{g}$ is isomorphic with the left-invariant differential operators; we keep the same notation for the vectors and the corresponding operators.

The dimension of $G$ is $n=\sum_{\ell} n_{\ell}$ while its homogeneous dimension is $Q=$ $\sum_{\ell} \ell n_{\ell}=v_{1}+v_{2}+\ldots+v_{n}$. 
We denote by $\widehat{G}$ the set of equivalence classes of (continuous) irreducible unitary representations of $G$. We will often identify a representation of $G$ with its equivalence class. We will also keep the same notation for the corresponding infinitesimal representation. For $\pi \in \widehat{G}$, we denote by $\mathcal{H}_{\pi}$ the representation space of $\pi$ and by $\mathcal{H}_{\pi}^{\infty}$ its subspace of smooth vectors. For $f \in L^{1}(G)$, we define its Fourier transform at $\pi \in \widehat{G}$ by $\widehat{f}(\pi)=\int_{G} f(g) \pi(g)^{*} d g$, with the integral understood in the Bochner sense. Denoting by $\mu$ the Plancherel measure on $\widehat{G}$, the inverse Fourier formula holds :

$$
f(g)=\int_{\widehat{G}} \operatorname{trace}(\pi(g) \widehat{f}(\pi)) d \mu(\pi) \quad \text { when } \quad \int_{\widehat{G}} \operatorname{trace}|\widehat{f}(\pi)| d \mu(\pi)<\infty .
$$

Let $\mathcal{R}$ be a positive (left) Rockland operator on $G$; this means that $\mathcal{R}$ is a leftinvariant differential operator, homogeneous of degree $\nu$ necessarily even, positive in the operator sense, and such that for every non-trivial $\pi \in \widehat{G}$ the operator $\pi(\mathcal{R})$ is injective on $\mathcal{H}_{\pi}^{\infty}$. The operator $\mathcal{R}$ admits an essentially self-adjoint extension on $C_{0}^{\infty}(G)$ (see [8]), and we will still denote this extension by $\mathcal{R}$. Examples of such operators are given in the stratified case by $\mathcal{R}=-\mathcal{L}$ where $\mathcal{L}=\sum_{1 \leq j \leq n_{1}} X_{i}^{2}$ is a Kohn-sub-Laplacian, and in the graded case by the operators

$$
\sum_{1 \leq j \leq n_{j}}(-1)^{\frac{\nu_{o}}{v_{j}}} X_{j}^{2 \frac{\nu_{o}}{v_{j}}} \text { and } \sum_{1 \leq j \leq n_{j}} X_{j}^{4 \frac{\nu_{o}}{v_{j}}},
$$

where $\nu_{o}$ denotes some common multiple of $v_{1}, \ldots, v_{n}$. In fact our class of operators do not depend on the choice of such an operator $\mathcal{R}$.

\section{REsults}

We aim at defining the symbol classes in terms of the operators $\mathcal{R}$ as above.

Definition 3.1. A symbol is a family of operators $\sigma=\{\sigma(x, \pi): x \in G,[\pi] \in \widehat{G}\}$, such that

1. for each $x \in G$, the family $\{\sigma(x, \pi), \pi \in \hat{G}\}$ is a $\mu$-measurable field of operators $\mathcal{H}_{\pi}^{\infty} \rightarrow \mathcal{H}_{\pi}$

2. there exist two constants $\gamma_{1}, \gamma_{2} \in \mathbb{R}$ such that for every $x \in G$, the operator $\pi(I+\mathcal{R})^{\gamma_{1}} \sigma(x, \pi) \pi(I+\mathcal{R})^{\gamma_{2}}$ is bounded on $\mathcal{H}_{\pi}$ uniformly in $\pi \in \hat{G}$;

3. for any $\pi \in \widehat{G}$ and any $u, v \in \mathcal{H}_{\pi}$, the scalar function $x \mapsto(\sigma(x, \pi) u, v)_{\mathcal{H}_{\pi}}$ is smooth on $G$.

Here, the powers $\pi(I+\mathcal{R})^{\gamma}$ are defined by the spectral theorem for the positive operator $\pi(\mathcal{R})$. The existence of $\gamma_{1}, \gamma_{2}$ in the second condition is used to guarantee that the following formula makes sense :

$$
T f(x)=\int_{\widehat{G}} \operatorname{trace}(\pi(x) \sigma(x, \pi) \widehat{f}(\pi)) d \mu(\pi) \quad, \quad f \in \mathcal{S}(G), x \in G ;
$$

indeed such operator $T=\operatorname{Op}(\sigma)$ is well-defined and continuous $\mathcal{S}(G) \rightarrow \mathcal{S}^{\prime}(G)$. We note that if the operator $T$ is left-invariant, then its symbol is independent of $x$.

Definition 3.2. The difference operators $\Delta^{\alpha}, \alpha \in \mathbb{N}_{0}^{n}$, are densely defined on the $C^{*}$-algebra of the group via

$$
\left(\Delta^{\alpha} \widehat{f}\right)(\pi):=\widehat{\left(x^{\alpha} f\right)}(\pi) \quad, \quad f \in \mathcal{S}(G) .
$$

Let now $m \in \mathbb{R}$ and $0 \leq \delta \leq \rho \leq 1$ with $\delta \neq 1$. 
Definition 3.3. The symbol class $S_{\rho, \delta}^{m}$ is defined as the set of symbols $\sigma$ satisfying for all $\alpha, \beta \in \mathbb{N}_{0}^{n}$ and every $\gamma \in \mathbb{R}$ :

$$
\sup _{x \in G, \pi \in \widehat{G}}\left\|\pi(I+\mathcal{R})^{\frac{\rho[\alpha]-m-\delta[\beta]+\gamma}{\nu}} X_{x}^{\beta} \Delta^{\alpha} \sigma(x, \pi) \pi(I+\mathcal{R})^{-\frac{\gamma}{\nu}}\right\|_{o p}<\infty .
$$

(The supremum over $\pi$ is in fact the essential supremum over the Plancherel measure $\mu$.)

It is easy to see that if $m_{1} \leq m_{2}, \delta_{1} \leq \delta_{2}$ and $\rho_{1} \geq \rho_{2}$, then $S_{\rho_{1}, \delta_{1}}^{m_{1}} \subset S_{\rho_{2}, \delta_{2}}^{m_{2}}$. Furthermore, the expressions in (3.1) define a Fréchet topology on the linear space $S_{\rho, \delta}^{m}$.

In the abelian case, that is, $\mathbb{R}^{n}$ endowed with the addition law and $\mathcal{R}=-\mathcal{L}, \mathcal{L}$ being the Laplace operator, $S_{\rho, \delta}^{m}$ boils down easily to the usual Hörmander class. However our initial motivation did not come from the abelian case : we wanted to define the difference operators and the symbol classes in analogy with the ones defined in [14] on compact Lie groups. In this case, a definition similar to (3.1) would formally give the same classes of symbols defined in 14 since, $\mathcal{R}=-\mathcal{L}, \mathcal{L}$ being the Laplace-Beltrami operator, the operator $\pi(I+\mathcal{R})$ is scalar. In our case, i.e. $G$ being a graded non-abelian Lie group, the operator $\mathcal{R}$ is not even central and the introduction of $\gamma$ in (3.1) assures that $\bigcup_{m \in \mathbb{R}} S_{\rho, \delta}^{m}$ is an algebra.

We have the following properties for the operators classes $\Psi_{\rho, \delta}^{m}:=\operatorname{Op}\left(S_{\rho, \delta}^{m}\right)$ defined using the quantisation procedure $\sigma \mapsto \mathrm{Op}(\sigma)$ described above.

Theorem 3.4. Let $0 \leq \delta \leq \rho \leq 1$. We have the following properties :

(1) The symbol classes is an algebra of operators $\bigcup_{m \in \mathbb{R}} S_{\rho, \delta}^{m}$ stable by taking the adjoint. Each vector space $S_{\rho, \delta}^{m}$ does not depend on the choice of the positive Rockland operator $\mathcal{R}$.

(2) For $\rho \neq 0$, the operator class $\bigcup_{m \in \mathbb{R}} \Psi_{\rho, \delta}^{m}$ is an algebra stable by taking the adjoint.

(3) For any $\alpha \in \mathbb{N}_{0}^{n}$, we have $X^{\alpha} \in \Psi_{1,0}^{[\alpha]}$.

(4) For any positive Rockland operator of homogeneous degree $\nu$, we have $(I+$ $\mathcal{R})^{\frac{m}{\nu}} \in \Psi_{1,0}^{m}$.

(5) If $\rho \in[0,1)$ then the operators in $\Psi_{\rho, \rho}^{0}$ are continuous on $L^{2}(G)$.

(6) Let $\rho \neq 0$. The integral kernel $K(x, y)$ of an operator $T \in \Psi_{\rho, \delta}^{m}$ is smooth on $(G \times G) \backslash\{(x, y): x=y\}$. It is of Calderón-Zygmund type in the sense of Coifman and Weiss [5, ch.III]. It decreases rapidly as $\left|x y^{-1}\right| \rightarrow \infty$ (here we have fixed a homogeneous norm $|\cdot|$ on $G$, i.e. a continuous function, homogeneous of degree one and vanishing only at 0$)$ : i.e. for any $M>0$ there exists $C_{M}>0$ such that

$$
\left|x y^{-1}\right| \geq 1 \Longrightarrow|K(x, y)| \leq C_{M}\left|x y^{-1}\right|^{-M} .
$$

At the diagonal it satisfies

$$
\left|x y^{-1}\right| \leq 1 \Longrightarrow|K(x, y)| \leq C\left|x y^{-1}\right|^{-\frac{Q+m}{\rho}} .
$$

By [5, ch.III théorème 2.4] Property (6) implies that the operators in $\Psi_{\rho, \delta}^{0}, 1 \geq$ $\rho \geq \delta \geq 0, \rho \neq 0, \delta \neq 1$, are continuous on $L^{p}(G), 1<p<\infty$.

By Properties (2) and (4), any operator in $\Psi_{\rho, \delta}^{m}, 1 \geq \rho \geq \delta \geq 0, \rho \neq 0, \delta \neq 1$, is continuous on the natural Sobolev spaces (denoted by $L_{a}^{2}(G)$ ) associated with the dilations and the loss of derivatives is controlled by the order $m$. The Sobolev 
space $L_{a}^{2}(G)$ is defined as the set of tempered distribution $f \in \mathcal{S}^{\prime}(G)$ such that $(I+\mathcal{R})^{\frac{a}{\nu}} f \in L^{2}(G)$ but does not depend on the choice of $\mathcal{R}$. These Sobolev spaces enjoy properties similar to the stratified case proved by Folland [7, in particular for interpolation (see [6]).

We now give the sharp Gårding inequality.

Theorem 3.5. Let $0 \leq \delta \leq \rho \leq 1, \rho \neq 0, \delta \neq 1$, and let $T \in \Psi_{\rho, \delta}^{m}$ with symbol $\sigma=\{\sigma(x, \pi)\}$. Assume that each $\{\sigma(x, \pi)\}$ of $T$ is non-negative on $\mathcal{H}_{\pi}$ (in the operator sense). Assume also that there exists a Rockland operator $\mathcal{R}$ such that each $\sigma(x, \pi)$ commutes with the spectral measure of $\pi(\mathcal{R})$ for every $x \in G$ and almost every $\pi \in \widehat{G}$. Then there exists $C>0$ such that for every $f \in \mathcal{S}(G)$ we have

$$
\operatorname{Re}(T f, f)_{L^{2}(G)} \geq-C\|f\|_{\frac{L_{\frac{m-(\rho-\delta)}{2}}^{2}}{}(G)} .
$$

The class includes

- the variable coefficient Kohn-sub-Laplacians and Rockland operators of the form $a(x) \mathcal{R}$, with $a(x) \geq 0$ satisfying $X^{\alpha} a \in L^{\infty}(G)$ for all $\alpha \in \mathbb{N}_{0}^{n}$,

- the multipliers $\phi(\mathcal{R})$ for a smooth function $\phi:[0, \infty) \mapsto[0, \infty)$ satisfying

$$
\forall a \in \mathbb{N}_{0} \quad \exists C=C_{a}>0 \quad \forall \lambda \geq 0 \quad\left|\partial_{\lambda}^{\alpha} \phi(\lambda)\right| \leq C(1+\lambda)^{\frac{m}{\nu}-a},
$$

- more generally the operators with symbols given by $\sigma(x, \pi)=\phi_{x}(\pi(\mathcal{R}))$ with $(x, \lambda) \mapsto \phi_{x}(\lambda)$ being non-negative and smooth on $G \times[0, \infty)$, and $\phi_{x}$ satisfying (3.2) at each $x$ with a constant $C$ independent of $x$.

The condition on the commutation with the spectral measure of $\pi(\mathcal{R})$ seems to be reasonable : in the corresponding version of the sharp Gårding inequality on compact Lie groups in [13] or in the abelian case, this condition is automatically satisfied there because if $\mathcal{R}=-\mathcal{L}$ and $\mathcal{L}$ is the Laplacian then $\pi(\mathcal{R})$ is central.

In [9, Helffer and Nourigat proved that $\mathcal{R}$ and, equivalently, $I+\mathcal{R}$ are hypoelliptic. We finally give the result stating that the Schwartz version of such hypoellipticity is also true.

Theorem 3.6. The operator $I+\mathcal{R}$ is Schwartz-hypoelliptic, i.e. for $f \in \mathcal{S}^{\prime}(G)$, the condition $(I+\mathcal{R}) f \in \mathcal{S}(G)$ implies $f \in \mathcal{S}(G)$.

In fact, our symbolic calculus allows the construction of a parametrix for the operator $I+\mathcal{R}$ from which both the hypoellipticity and the Schwartz hypoellipticity follow.

\section{ACKNOWLEDGEMENTS}

The first author acknowledges the support of the London Mathematical Society via the Grace Chisholm Fellowship. It was during this fellowship held at King's College London in 2011 that the work was initiated. The second author was supported in part by the EPSRC Leadership Fellowship EP/G007233/1.

\section{RÉFÉRENCES}

[1] Bahouri H., C. Fermanian-Kammerer and I. Gallagher, Phase-space analysis and pseudodifferential calculus on the Heisenberg group, Astérisque 342 (2012).

[2] R. Beals and P. Greiner, Calculus on Heisenberg manifolds. Princeton University Press, 1988.

[3] M. Christ, On the regularity of inverses of singular integral operators, Duke Math. J. 57 (1988), 459-484. 
[4] M. Christ, D. Geller, P. Glowacki and L. Polin, Pseudodifferential operators on groups with dilations, Duke Math. J. 68 (1992), 31-65.

[5] R. Coifman and G. Weiss Analyse harmonique non-commutative sur certains espaces homogènes, Springer, 1971.

[6] V. Fischer and M. Ruzhansky, Quantization on nilpotent Lie groups, monograph in preparation.

[7] G. B. Folland, Subelliptic estimates and function spaces on nilpotent Lie groups, Ark. Mat., 13 (1975), 161-207.

[8] G. B. Folland and E. Stein, Hardy spaces on homogeneous groups, Princeton University Press, 1982.

[9] B. Helffer and J. Nourrigat, Caracterisation des opérateurs hypoelliptiques homogènes invariants à gauche sur un groupe de Lie nilpotent gradué, Comm. Partial Differential Equations, 4 (1979), 899-958.

[10] A. Hulanicki, A functional calculus for Rockland operators on nilpotent Lie groups, Studia Math., 78 (1984), 253-266.

[11] R. Ponge, Heisenberg calculus and spectral theory of hypoelliptic operators on Heisenberg manifolds, Mem. Amer. Math. Soc. 194 (2008).

[12] M. Ruzhansky and V. Turunen, Pseudo-differential operators and symmetries : Background analysis and advanced topics, Birkhäuser, Basel, 2010.

[13] M. Ruzhansky and V. Turunen, Sharp Gårding inequality on compact Lie groups, J. Funct. Anal., 260 (2011), 2881-2901.

[14] M. Ruzhansky and V. Turunen, Global quantization of pseudo-differential operators on compact Lie groups, SU(2) and 3-sphere, Int Math Res Notices IMRN (2012), 58 pages, doi : 10.1093/imrn/rns122.

[15] M. E. Taylor, Noncommutative microlocal analysis. I. Mem. Amer. Math. Soc. 52 (1984).

[16] M. E. Taylor, Noncommutative harmonic analysis, American Mathematical Society, 1986.

Universita degli studi di Padova, DMmMSA, Via Trieste 63, 35121 Padova, Italy

E-mail address: fischer@dmsa.unipd.it

180 Queen's Gate, Department of Mathematics, Imperial College London, London, SW7 2AZ, United KINGDOM

E-mail address: m.ruzhansky@imperial.ac.uk 